\title{
La política de adelanto en cursos para repitentes en la educación secundaria,|Costa Rica: estudio de caso
}

\author{
Public policy about advanced courses to repeaters in secondary education, Costa Rica: case study
}

Recibido 20 agosto 2015 • Aceptado 08 octubre 2015 • Corregido 22 noviembre 2015

\author{
Allan Abarca Rodríguez' \\ Universidad de Costa Rica \\ San José, Costa Rica \\ allan.abarca@ucr.ac.cr
}

\begin{abstract}
Resumen. En el año 2009, se inicia en Costa Rica la ejecución de la reforma del sistema de promoción escolar en secundaria, aprobado en el año 2008 por el Consejo Superior de Educación: la persona estudiante que reprueba asignaturas, tiene el deber de matricularlas en un próximo año escolar, pero el derecho de cursar materias del siguiente nivel (para las cuales ya aprobó el requisito). Este artículo es producto de una investigación en la que se analizan los criterios de actores de un centro educativo, respecto a las repercusiones de la política pública en la práctica, sus aspectos positivos y negativos, y de la concepción general de la política, a partir de su vigencia. En total, se entrevistan 66 personas: 25 estudiantes que adelantan cursos, 25 docentes, 12 estudiantes de alto rendimiento académico y cuatro dirigentes gremiales. Entre otros aspectos, se concluye que se ha generado una alta complejidad académico-administrativa con repercusiones académicas no deseadas; esto implica que la alta legitimidad inicial de la política-considerada como justaesté en entredicho por las sensibles consecuencias negativas de su ejecución.
\end{abstract}

Palabras clave. Política pública; decisión; promoción escolar

\begin{abstract}
In 2009, in Costa Rica begins the execution of the secondary school promotion system's upgrade approved in 2008 by the Higher Education Council: Student who unapproved subjects have a duty to enroll in the next year but they have a right to register the subjects in the next level (for which already approved the requirement). This article is the result of a research in which analyze the opinion's actors in a secondary school about the effects of public policy in practice, its positive and negative aspects and public policy's general conception for its validity. In total, 66 people were interviewed: 25 students who advanced courses of them, 25 teachings, 12 students of high academic performance and 4 union leaders. On the other hand, it concludes that it generated a high academic
\end{abstract}

1 Magíster Scientiae en Gobierno y Asuntos Públicos (FLACSO-México). Magíster Scientiae en Salud Pública, egresado del Magíster Scientiae en Gerencia Social de Trabajo Social, Licenciado en Ciencias de la Educación, Licenciado en Ciencias Políticas, titulaciones recibidas en la Universidad de Costa Rica (UCR). Catedrático de la Universidad de Costa Rica (UCR). Es docente-investigador en la Escuela de Salud Pública de la Facultad de Medicina y en la Escuela de Ciencias Políticas de la Facultad de Ciencias Sociales, ambas en la UCR. Ha sido invitado a impartir docencia en la Maestría de Salud Pública, la Maestría Centroamericana de Ciencias Políticas y en la Maestría de Administración Universitaria de la Universidad de Costa Rica (UCR). 
and administrative complexity with unwanted academic impacts, this implies the policy's high inceptive legitimacy -considered fair- is in issue by execution's sensitives negatives consequences.

Keywords. Public policy; decision; school promotion

\section{Introducción}

En el año 2008, el Consejo Superior de Educación (CSE) reforma las políticas de promoción en educación secundaria, siendo la más significativa aquella que avala que una persona estudiante repitente pueda matricular asignaturas en niveles superiores, si es que el año anterior aprobó la respectiva asignatura par; por ejemplo, si es repitente en el año $x$, podrá matricular la asignatura 'Matemática' del nivel superior, si es que en el año $x-1$ aprobó el requisito².

Actores involucrados han denominado a esa política pública como la posibilidad de adelanto de cursos o la política de arrastre de cursos. En algunos centros educativos, nominan a los que adelantan cursos como estudiantes de avance.

Se realiza un estudio de caso a partir de la escogencia no arbitraria de un centro educativo de secundaria. La investigación supuso que la política de adelantamiento podría tener repercusiones diferentes según el centro educativo fuese de baja o alta matrícula. Para esto, se manipula la megabase de datos producida por el Tercer Informe del Estado de la Educación (Programa Estado de la Educación, 2011) -que a su vez amalgama más de 70 bases de datos dispersas del Ministerio de Educación Pública (MEP) sobre diversos indicadores de los centros educativos del país, introduciéndole ajustes para la obtención de datos fiables-.

Se procede a seleccionar los centros educativos, para los cuales haya datos para los tres años previos a la ejecución de la reforma en estudio $(2006,2007$ y 2008) y un año con la reforma en ejecución (2009); a partir de esos datos, se formula un índice considerando las tasas de repitencia y deserción, desagregando por centros de alta matrícula y de baja matrícula.

De esta forma, se elige al Liceo Virgen de la Medalla Milagrosa, centro educativo calificado de baja matrícula-se ubica en los dos primeros deciles de matrícula del sistema educativo-y que a su vez, se posiciona en la lista de los 20 centros educativos con mayor tasa en deserción y en repitencia (a nivel de sétimo año), según el índice formulado en la investigación, para el período señalado.

El centro educativo seleccionado es de fácil acceso geográfico -se ubica en Vista de Mar de Goicoechea, en el Valle Central-, y pertenece al distrito de Ipís, de relativo bajo nivel de desarrollo, cuya población con una carencia o más se sitúa alrededor del 14\% (Instituto Nacional de Estadística y Censo [INEC], 2013).

2 Este artículo se basa en los resultados parciales de la investigación Análisis de la política pública de reforma integral de las normas de promoción y repitencia dentro del sistema educativo costarricense, inscrito en la Vicerrectoría de Investigación de la Universidad de Costa Rica (N. 213-B1-322). 


\section{Participantes y métodos de investigación}

En ese centro educativo se realizó contacto con tres tipos de sujetos:

a. Estudiantes repitentes de sétimo año que adelantaban materias de octavo nivel en el año 2012 (se les abordó en el año 2012 dos veces y en el 2013 en una ocasión). Se les califica como estudiantes de avance. Mediante entrevista estructurada se les aplica un cuestionario.

b. Estudiantes de alto rendimiento académico de octavo y de noveno nivel (año 2013). La técnica que se aplica es la de grupos focales.

c. Docentes activos (año 2013). Se abordan mediante entrevista estructurada.

d. Los y las estudiantes de avance tenían las siguientes condiciones:

- Reprobaron uno o más cursos en el año lectivo 2011.

- Repiten cursos reprobados y adelantan cursos en el año 2012.

En mayo del año 2012, se recopilan datos de los 25 estudiantes que constituían el universo de estudiantes de avance; se les aborda en dos ocasiones adicionales (tercera semana de agosto de 2012 y noviembre de 2013), repitiendo algunos ítems de un cuestionario estructurado, sobre todo para constatar el cambio o la permanencia en algunos criterios.

Se gestionaron y realizaron dos grupos focales con estudiantes de alto rendimiento académico, un grupo de estudiantes matriculados en octavo año y otro en el noveno año.

El interés de tener contacto con esta población, se da a partir de dos presunciones:

a. Su destacado rendimiento y alta asistencia a clases les hace percibir con claridad las opiniones propias-o de otros pares-respecto de los compañeros estudiantes que adelantan.

b. Ningún estudio conocido sobre la temática ha tomado en cuenta la opinión de los estudiantes que aprueban con regularidad los años escolares y que lo hagan con alto desempeño académico.

Los dos grupos focales permiten obtener información respecto de:

a. Criterio acerca de la pertinencia o no de la política pública.

b. Percepción sobre los estudiantes de avance con quienes han compartido en el aula.

Se entrevistan a 25 docentes (de los cuales el 68\% también labora en al menos otro centro educativo de secundaria). Se aplica un cuestionario estructurado que contiene preguntas sobre cuatro tópicos generales: 
a. Experiencia sobre el adelanto de cursos.

b. Criterio sobre las normas de promoción anteriores (las reemplazadas).

c. Opinión sobre la continuidad de la política.

d. Posibles cambios respecto de la política de promoción.

Finalmente, se procedió a entrevistar a dirigentes gremiales del sector educativo acerca del origen, fundamento y repercusiones de la decisión de la política educativa en cuestión:

a. Presidente de la Asociación Nacional de Educadores (ANDE).

b. Presidenta Asociación de Profesores de Segunda Enseñanza (APSE).

c. Presidente Sindicato de Trabajadores de la Educación Costarricense (SEC).

d. Presidente Colegio de Licenciados y Profesores en Letras, Filosofía, Ciencias y Arte (COLYPRO).

Los resultados de las entrevistas y grupos focales se procesaron con el programa informático SPSS, versión 22 y en el ATLAS.ti, versión 7.

\section{Referente teórico}

Una política pública es la respuesta de la institucionalidad pública -aunque también puede emanar de una Organización No Gubernamental (ONG) o una empresa privada-, ante un asunto que se considera un problema público -real o potencial- a intervenir. Trasciende un simple enunciado de voluntad -v. g. - disminuir la deserción escolar- - sino que - exige un proceso racional, informado y comprometido de selección y definición de problemas, a partir de los medios efectivamente disponibles para solucionarlos- (Merino, 2013, p. 36).

En la diversidad conceptual expuesta por autores y autoras, se precisan los siguientes elementos comunes: "objetivos definidos, cursos de acción, un problema público, y el apoyo de diversas organizaciones en su ejecución" (Holguín, 2013, p. 176). En forma complementaria, hay diversos aspectos que se pueden considerar para establecer el blindaje con que fueron formuladas -y que el Banco Interamericano de Desarrollo (BID) las considera como características-, entre ellas: "la estabilidad, la adaptabilidad, la coherencia, la calidad de la implementación, la orientación hacia el interés público y la eficiencia" (BID, 2006, p. 17).

Un aspecto presente en la evaluación de una política en curso, refiere a cómo las y los actores entienden e incorporan esa política en su rutina diaria. Una política pública cumplirá más difícilmente sus objetivos, si ocurre una o varias de las siguientes situaciones: 
a. La decisión emanada mediante una instrucción implica a varios actores.

b. Si los procedimientos nuevos cambian un elevado porcentaje de las rutinas administrativas.

c. No hay incentivos acordes.

d. Se genera una acumulación de procedimientos que pueden ser diversos y contradictorios en medio de recursos escasos para cumplirlos de la mejor forma. (Merino, 2013, pp. 143-144)

\section{Resultados y análisis de la información}

\section{La política de adelanto desde lo justo}

Consultados de si la decisión del MEP - de dejarlos matricular materias de otro nivel siendo repitentes- es justa o no, una gran mayoría del estudiantado de avance se mostró a favor (siete de cada 10); curiosamente un $16 \%$ se muestra "en desacuerdo", aunque no hay uno solo en "total desacuerdo" (véase Figura 1).

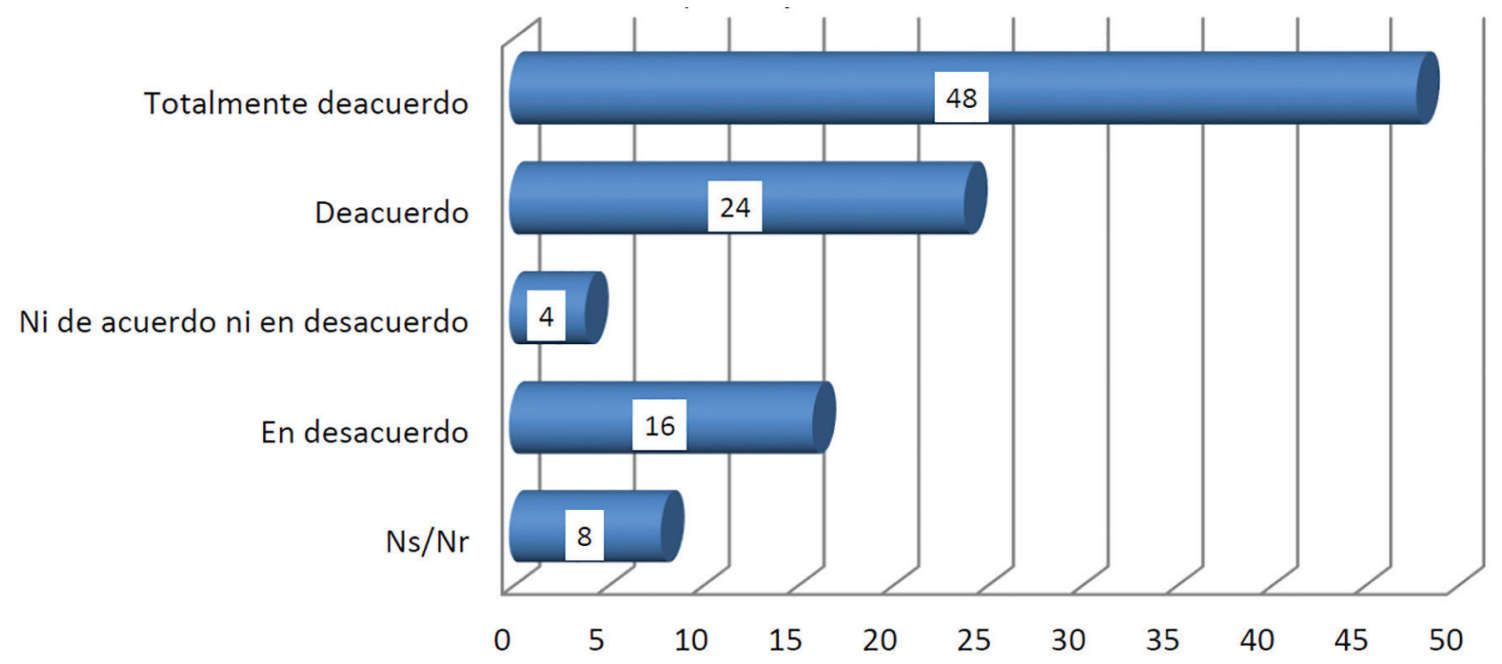

Figura 1. Criterio de estudiantes de avance, Liceo Virgen de la Medalla Milagrosa (2012), respecto a lo justo de la política de adelantamiento de cursos

Por su parte, las y los estudiantes de excelencia académica puntualizan dos aspectos positivos de la política de adelantamiento: 
a. En general se concibe como "justa", particularmente para estudiantes que reprueban solamente un curso o dos.

b. En sí misma, se concibe como un estímulo para "no retrasarse" en los estudios.

Aunque hay que recalcar que el estudiantado de excelencia no deja de señalar repercusiones negativas de la política, sobre todo cuando hay una tendencia mayoritaria de calificar al estudiantado de avance como "poco esforzados" y "generadores de distracción" para el resto de estudiantes.

En forma complementaria, se le consulta al personal docente sobre lo justo o no de algunas normas de aprobación anteriores, que incluso motivaron la reforma de promoción. Específicamente se les consultó acerca de tres afirmaciones:

a. Era injusto que si un estudiante reprobaba en tres materias que debiera cursar todas las materias del nivel (año) en que se encontraba.

b. Era injusto que si un estudiante debía aprobar con 65 o 70 el promedio anual de notas, que las notas del tercer trimestre también deberían alcanzar 65 o 70 como mínimo.

c. Era injusto que si un estudiante tenía en conducta un promedio anual menor al necesario para aprobar, estaba obligado a presentar examen en todas las otras asignaturas, cuyo promedio fuese menor a 80.

\section{Tabla 1}

Criterios del personal docente sobre lo injusto o justo de ciertas normas de promoción previas a las reformas del año 2008, Liceo Virgen de la Medalla Milagrosa (2013) -en porcentaje-

\begin{tabular}{|c|c|c|c|}
\hline AFIRMACIÓN & $\begin{array}{l}\text { De acuerdo } \\
\text { o muy de } \\
\text { acuerdo }\end{array}$ & $\begin{array}{l}\text { Ni de } \\
\text { acuerdo ni en } \\
\text { desacuerdo }\end{array}$ & $\begin{array}{c}\text { En desacuerdo } \\
\text { o muy en } \\
\text { desacuerdo }\end{array}$ \\
\hline Es injusto que se deban repetir todas las materias. & 40 & 28 & 32 \\
\hline $\begin{array}{l}\text { Es injusto que incluso en las notas del tercer trimestre el } \\
\text { estudiante deba alcanzar un mínimo para aprobar }\end{array}$ & 44 & 8 & 48 \\
\hline $\begin{array}{l}\text { Es injusto que si en conducta el promedio anual no } \\
\text { es aprobable se deba presentar examen en todas las } \\
\text { asignaturas con promedio menor a } 80\end{array}$ & 48 & 20 & 32 \\
\hline
\end{tabular}

Nota: Construcción propia a partir de la información suministrada. 
Aunque casi la mitad del personal docente califique de injustas algunas normas de promoción escolar precedentes, también es interesante constatar que un gran segmento las apoyaba o al menos tienen dudas de si eran injustas. Particularmente, un tercio del personal docente no cree que sea injusto que un sistema educativo obligue a repetir cursos que ya se habían aprobado.

\section{La política de adelanto desde las repercusiones administrativas y académicas}

Consultado el estudiantado de avance respecto de las desventajas -en caso que las hubiere- de adelantar cursos de otro nivel, hay un señalamiento generalizado en tres aspectos:

a. Los tiempos extensos que deben esperar cuando salen de una clase ubicada en un nivel para ingresar a una clase ubicada en otro nivel (88\%).

b. La espera para ingresar a un curso de adelanto se hace sin tener las condiciones adecuadas para estudiar o realizar tareas (88\%).

c. La imposibilidad de matrícula-adelantamiento-de todas las materias que se desearía, por choque de horarios (60\%).

El propio estudiantado de alto desempeño académico -que se aborda mediante grupos focales- indica factores externos que a su criterio reducen la efectividad de la política:

a. Las y los estudiantes que adelantan están expuestos a constantes cambios de horarios, lo cual atenta contra una lógica de planificación y asistencia a las clases.

b. Los tiempos de espera entre lecciones son muy extensos, y no hay áreas de estudio acordes para esa condición.

Por otra parte, procesando las entrevistas realizadas a dirigentes de gremios sindicales, en el programa informático ATLAS.ti, destaca la presunción unánime de calificar a la política como una "decisión inconsulta" y de una "decisión justa pero no técnica". Este último punto, refiere a que si bien había aspectos de la política de promoción previa al 2008 que eran equívocas -por ejemplo que estudiantes que han aprobado cursos deben repetirlas-, la decisión propiamente de "adelantamiento" se presume errónea por las consecuencias que implica (véase Figura 2). 


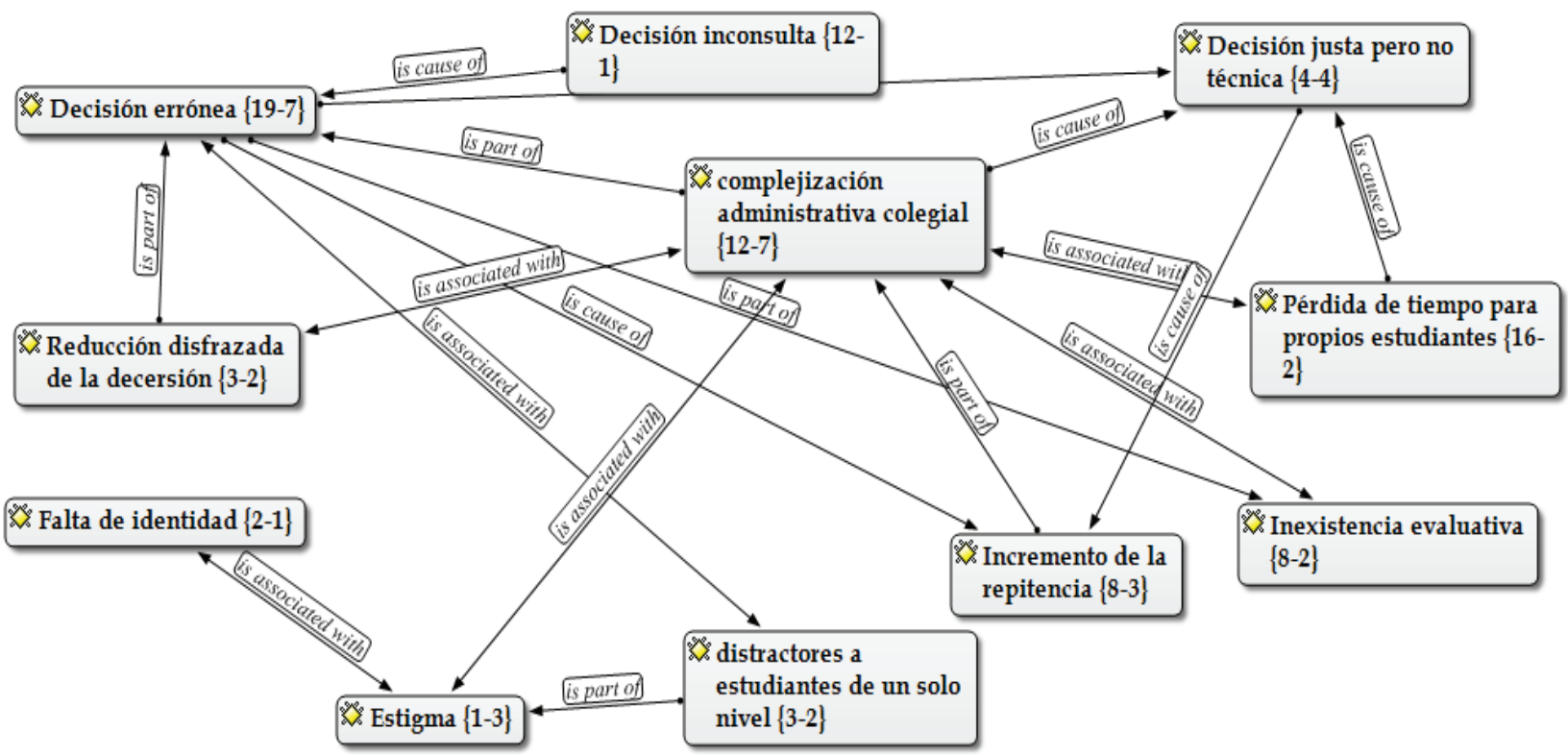

Figura 2. Red de categorías de análisis, criterios de dirigentes gremios sindicales respecto de la política de adelantamiento de cursos (2012)

Nota: Construcción propia basada en entrevistas realizadas y procesamiento con ATLAS.ti

En la posición de los gremios hay una insistencia en catalogar a la política en cuestión como una "decisión unilateral" y "decisión errónea", siendo la principal repercusión la complejidad de la "administración general" que se debe realizar desde el centro educativo ${ }^{3}$. Hay una suerte de bola de nieve que impacta en consecuencias negativas, principalmente en el estudiantado que adelanta, y del propio ajuste didáctico y de tiempos que debe realizar cada docente.

Las consecuencias apuntadas por los gremios son de dos tipos:

a. Las inmediatas o visibles: estudiantes en pasillos, distractores en clases, estigmatización, falta de identidad, pérdida de tiempo para los propios estudiantes que adelantan, complejidad administrativa colegial.

b. Las no visibles: aumento de los niveles de repitencia, reducción disfrazada de la deserción.

3 Para una discusión amplia sobre el marco decisional colegiado de esta política pública, cfr. Picado, C. y Abarca, A. (2012). La política pública en promoción y repitencia en la Educación Secundaria, Costa Rica, Anuario Centro de Investigaciones y Estudios Políticos, 3, 126-147. 
Se da el criterio que el "adelantamiento" es falso porque hay una tendencia de estar matriculado en varios niveles, que en el corto plazo se presume una baja en la tasa de la deserción pero que en el fondo es, más bien, una consolidación de la repitencia. El otro aspecto interesante es que si bien se asume "lo inconsulto" de la política, también hay una insistencia en que las autoridades educativas no han iniciado una evaluación oficial o formal de las implicaciones de la política en todas sus dimensiones.

Al respecto, incluso la propia Contraloría General de la República (2011) ha señalado que, en la aprobación de la propuesta, "no medió "evidencia técnica" que despejara los acontecimientos que se preveían" (pp. 18-19).

\section{Otro tipo de consecuencias}

Se ha discutido acerca de las posibles disfuncionalidades que produce la política de adelantamiento, particularmente de la dificultad de integración del estudiante -situado al menos en dos niveles distintos-y la emergencia de estereotipos. Al respecto, el $80 \%$ del personal docente afirma que "el/la estudiante que adelanta cursos enfrenta problemas de identidad al nivel al que pertenecen".

En la primera entrevista realizada con el estudiantado de avance-iniciando el curso lectivoel 75\% dijo encontrarse "debidamente aceptado e integrado en los dos niveles". No obstante, en la tercera interacción se les consulta su grado de acuerdo con la siguiente afirmación: "por lo general un o una estudiante que adelanta cursos enfrenta problemas de integración con sus compañeros"; al respecto, el $40 \%$ responde afirmativamente, un $30 \%$ se manifiesta en contra de esa idea y el 30\%, manifiesta ni estar en desacuerdo ni de acuerdo.

La mayoría del estudiantado (68\%) ha escuchado la frase que "la opción de adelantar es una vagabundería". Esa expresión la ha escuchado de sus padres, el 20\%, y de sus compañeros y compañeras, el $30 \%$.

Otro elemento que entra a discusión es acerca del compromiso académico del estudiantado que avanza. La mayoría del personal docente consultado es del criterio que la política pública de adelanto, en general, beneficia a estudiantes de rendimiento deficiente, incluso el 92\% está de acuerdo o muy de acuerdo en la afirmación que la política de adelantamiento de cursos produce conformismo en el estudiantado (véase Figura 3). 
La política beneficia, en general, a los malos estudiantes

El derecho de adelantar cursos genera conformismo en los estudiantes

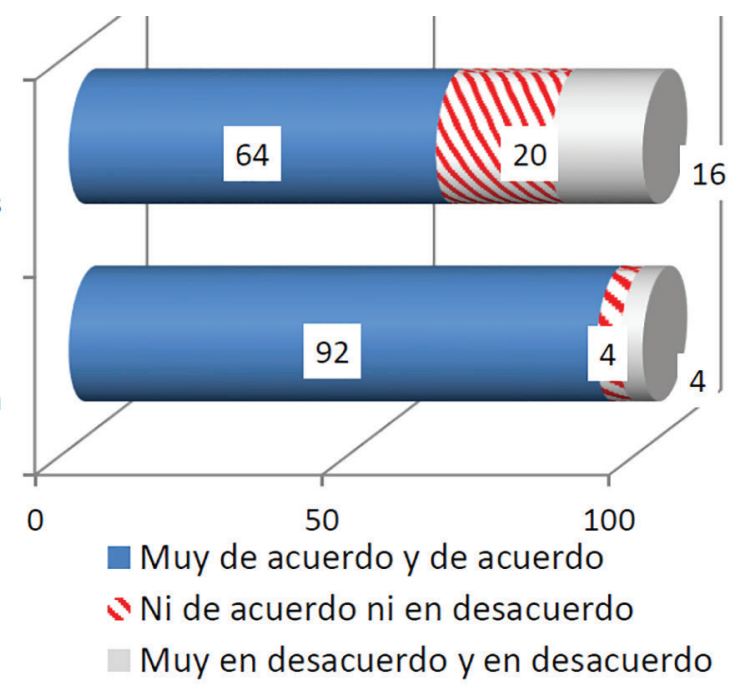

Figura 3. Criterios del personal docente, Liceo Virgen de la Medalla Milagrosa (2012), respecto de la política de adelantamiento de cursos -en porcentaje-

Por su parte, el estudiantado de rendimiento académico sobresaliente califica, en su mayoría, como "malos estudiantes" a su par de "avance". Incluso asimilan la estancia compartida del aula como una externalidad negativa para su proceso de enseñanza-aprendizaje. Aunque también hay que decir que reconocen que, cuando hay estudiantes repitentes de un solo curso, por lo general, tienen una percepción muy buena, calificando el acto reprobatorio de "accidente académico" y que -como se dijo más arriba- la posibilidad de adelantar materias "es justo".

Hay múltiples nodos relacionados -como categorías centrales inmersas en los criterios esgrimidos por las y los buenos estudiantes-; entre ellos, que el estudiantado de avance, en general, tienen menor rendimiento académico respecto del promedio y mayor irresponsabilidad. Este último -la irresponsabilidad- la notan, por ejemplo, en aspectos como que los docentes les llaman constantemente la atención, no llevan las tareas encomendadas, entre otros (véase Figura 4). 


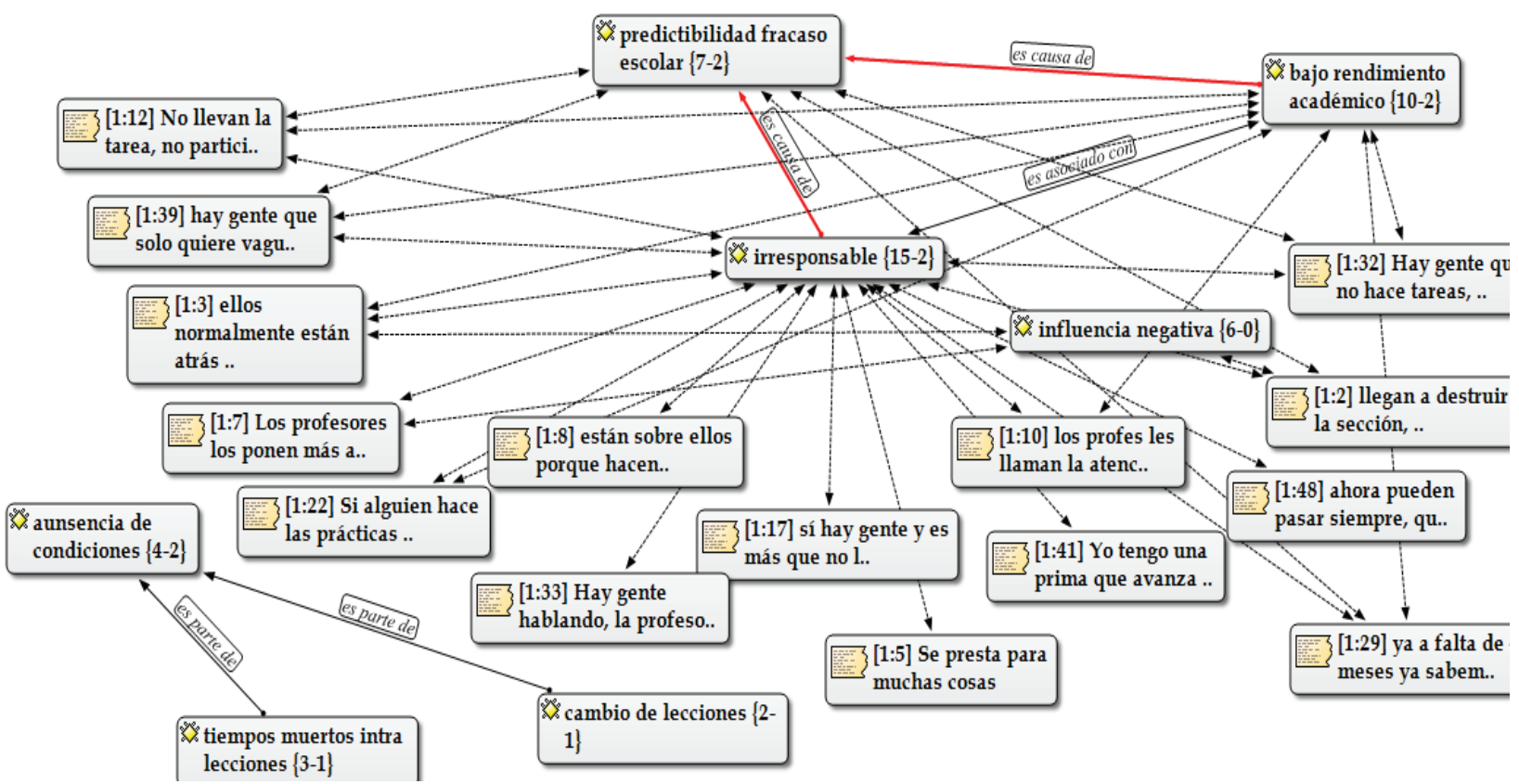

Figura 4. Red de categorías de análisis de criterios de estudiantes de rendimiento escolar alto respecto de la política de adelantamiento de cursos, Liceo Virgen de la Medalla Milagrosa (2013)

Nota: Construcción propia basada en grupos focales y el procesamiento con ATLAS.ti

A los estudiantes de avance se les pregunta su grado, respecto de la afirmación: "el derecho de adelantar cursos genera conformismo en los estudiantes". El 50\% manifiesta algún grado de acuerdo, el $40 \%$ no está de acuerdo ni en desacuerdo con la afirmación; el 10\% muestra algún grado de desacuerdo.

Incluso el $20 \%$ cree que "la medida de permitir 'adelantamiento' de cursos, en general, beneficia a malos estudiantes", mientras que un $40 \%$ ni apoya ni contradice la aseveración.

Dada la idea presente en docentes y estudiantes de excelencia respecto que el estudiantado de avance es proclive a retirarse de los cursos que adelantan, en la segunda interacción con tales estudiantes se les preguntó en ese sentido. El reactivo fue: "en este momento del año escolary de acuerdo a como le está yendo tanto en los cursos de sétimo como de octavo, ¿qué cree que es más importante, aprobar las materias de sétimo año, las de octavo año o ambas?" 
Se les aplicó tres alternativas para que eligieran; los resultados son los siguientes:

- $\quad 88 \%$ indica: "A como están mis notas hasta hoy día, creo que es más importante aprobar las materias de sétimo año".

- $12 \%$ indica: "A como están mis notas hasta hoy día, creo que es tan importante aprobar las materias de sétimo como las de octavo año".

- $0 \%$ indica: "A como están mis notas hasta hoy día, creo que es más importante aprobar las materias de octavo año".

Se afirma que, conforme se desarrolla el año escolar, hay un interés mayor en la aprobación de los cursos que se repiten que aquellos que se adelantan.

\section{Balance}

Consultados y consultadas en el primer contacto sobre "¿qué es lo bueno o positivo de la posibilidad de matricular materias de un nivel siguiente?", el estudiantado de avance dio 27 razones.

En principio, las respuestas no arrojan una opinión dominante sobre los beneficios de la posibilidad de adelanto, desde la óptica del estudiantado. A pesar que la pregunta abierta dio la libertad de escribir hasta tres razones, hubo dos personas que dejaron esa pregunta en blanco, incluso cinco dijeron que esa posibilidad de adelanto "no tiene nada de bueno" y cinco escribieron "no sé" (véase Tabla 2).

\section{Tabla 2}

Criterios de estudiantes sobre lo positivo de adelantar materias, Liceo Virgen de la Medalla Milagrosa (2012), -números absolutos-

\begin{tabular}{l|l}
\hline Argumento & Frecuencia \\
\hline "Avanzar materias de 8avo". & 5 \\
\hline $\begin{array}{l}\text { "Que no me obligan a matricular materias que ya había } \\
\text { aprobado". }\end{array}$ & 5 \\
\hline “No tiene nada de bueno". & 5 \\
\hline "No sé". & 5 \\
\hline "Acompañar a los amigos del año anterior". & 3 \\
\hline "Aprender más". & 2 \\
\hline Sin respuesta. & 2
\end{tabular}

Nota: Construcción propia a partir de los datos suministrados. 
Consultado el personal docente si el hecho que estudiantes repitentes matriculen cursos del siguiente nivel "afecta negativamente a los(as) estudiantes del nivel superior que sí llevan el bloque completo de cursos", el 52\% cree que sí se da una afectación, mientras tanto el $36 \%$ cree que no lo hay, frente a un $12 \%$ que no sabe o prefiere no responder.

Las justificaciones señaladas como de "afectación a los estudiantes del nivel superior", se pueden agrupar en tres criterios generales:

a. Interrumpen a los otros estudiantes (generan distracciones).

b. Manifiestan una falta de interés en el curso que adelantan.

c. Los pares no los toman en cuenta como integrantes del grupo.

Algunas de las frases expresadas son las siguientes:

- "Porque muchos de los alumnos que adelantan no les interesa la lección que se imparte e interrumpen constantemente"

- "Generalmente los estudiantes repitentes tienen una mala actitud y generan distractores a los otros compañeros"

- "Porque cuando uno pasa lista y el muchacho no está en clase, el nivel superior contesta 'él arrastra', y se forman estereotipos"

Una de las razones esgrimidas por el MEP es que la posibilidad de adelantamiento o avance de cursos hace que el o la estudiante sea menos proclive a la deserción (CSE, 2008; MEP, 2011). No obstante, en la primera interacción con el estudiantado de avance, se les consulta al respecto, obteniéndose un porcentaje bajo que la sitúa como la principal razón:

- Solamente el $12 \%$ opina que la oportunidad de "adelantar materias" es la razón principal por la cual no se fue del colegio

- El 12\% asegura que la oportunidad de "adelantar materias es una razón, entre muchas otras", por la que no se fue del colegio

- El 32\% opina que la oportunidad de "adelantar materias" es beneficiosa, pero que "no fue la razón por la que no me fui del colegio"

Resalta el hecho de que sea muy bajo el porcentaje que asegura que la posibilidad de adelantar materias sí ha sido razón para no haber abandonado el colegio. También, es curioso que el $44 \%$ no sabe qué responder - de si la posibilidad de adelanto contribuye a que no deserten-. La razón es que estos estudiantes no ven las normas como "un cambio", dado que 
ingresaron al sistema colegial con esas reglas; en otras palabras, un reactivo más pertinente debió ser: "si se le negara la posibilidad de adelantar materias del siguiente nivel, ¿se hubiera ido del colegio?"

\section{Conclusiones y recomendaciones}

- Hay un rechazo mayoritario por parte del personal docente sobre la política de promoción académica que permite al estudiantado repitente adelantar cursos del siguiente nivel. Esta visión es compartida por la dirigencia sindical, quienes califican a la política como inconsulta y no planeada.

- La política pública que instituye los cambios de promoción -entre ellos el de avance o adelanto- encontró viabilidad en un cuerpo colegiado (CSE) en virtud de la legitimidad propia de la política -tanto detractores como beneficiarios coinciden mayoritariamente en que es justa- pero que en la práctica encuentra muchas repercusiones negativas (afectación del entorno administrativo y de las propias condiciones de quienes están presentes en el acto educativo en el aula) y efectos positivos reducidos. Se está en presencia de una política pública con aparente alta legitimidad pero que en términos de eficiencia y de eficacia aún tiene que estudiarse.

- El cuerpo docente y el estudiantado de excelencia, en general, tienen visiones mayoritariamente negativas sobre el rendimiento y el compromiso académico del estudiantado que adelanta. No obstante, los estudiantes de excelencia consideran la política en sí como justa -mismo criterio de quienes adelantan-, y la creen conveniente, particularmente con estudiantes que repiten tan solo uno o dos cursos.

- El estudiantado de avance es crítico respecto a los tiempos extensos que debe esperar -sin condiciones para el aprovechamiento académico- para ingresar a clases ubicadas en distinto nivel; además, juzga como limitada la oferta de matrícula de adelanto a la que tiene derecho. Esa visión es compartida por el propio estudiantado de alto desempeño académico; este último añade que al inicio del ciclo lectivo es habitual el cambio de horario, lo que de por sí incide en una asistencia irregular por parte de sus "compañeros de adelanto" en las dos primeras semanas de lecciones, las cuales son fundamentales para generar empatía, compromiso y evitar el rezago en responsabilidades académicas.

- Hay suficiente evidencia para sospechar de la falta de rigurosidad de la matrícula de quienes adelantan. Esto es, pareciera que el proceso de "desvincularse de la matrícula que se adelanta" es relativamente fácil de realizar, a partir del momento en que el o la estudiante cataloga como prioridad la aprobación de las materias del nivel inferior o una incomodidad manifiesta en adelantar cursos. En otras palabras, hay que generar las estadísticas fiables de: a. aceptación de la inclusión en un grupo de avance (solicitudes 
aceptadas) y b. retiros aceptados por parte de la institución de los "avances" gestionados.

- El cuerpo docente cree que con algunas condiciones podría darse una política de promoción distinta para aquellos estudiantes que reprueben un solo curso en el año escolar, y con ello estimular la posibilidad de aprobación y que no provoque el retraso del estudiante (que reprueba una sola materia). Debe generarse un estudio sobre nuevas políticas de promoción que en sí ataquen las causas principales aducidas por el MEP, para apoyar la reforma del 2008 (evitar retrasos significativos en el estudiantado que pierde uno o pocos cursos, y que un(a) estudiante repitente no tenga que cursar los ya aprobados). Pero esa búsqueda de nuevas opciones debería de darse en un marco general mayor, la de una discusión sobre la mejora de lo que ha sido la política vigente en la práctica: sus elementos beneficiosos y negativos.

- Debe darse una investigación que enfoque el rendimiento académico del estudiantado de adelanto y verificar -mediante un seguimiento- si en vez de una evitabilidad de la deserción lo que se está dando es una postergación de ese evento. Como correlato, es interesante determinar el tiempo promedio de espera entre cursos y la aprovechabilidad de ese tiempo para el estudio por parte del estudiante, ya que, al menos en el centro educativo abordado, la infraestructura disponible imposibilita espacios para el estudio o la realización de tareas, lo cual es una contradicción, ya que el ejercicio del derecho de adelantar se da con pocas condiciones favorables.

- En general, la gestión de los centros educativos respecto de la planificación horaria de los cursos se vincula con la restricción de nombramientos del personal docente que labora en otros centros educativos, por ejemplo el $68 \%$ del cuerpo docente abordado al menos laboraba en otro centro educativo. La vulnerabilidad administrativa y académica pareciera ser mayor para centros educativos de baja matrícula, aspecto que debe estudiarse, pues disminuye las posibilidades de matrícula de cursos de adelanto $y$, por ende, hace susceptibles a los estudiantes de prolongadas esperas para asistir a la clase respectiva.

\section{Referencias}

Banco Interamericano de Desarrollo (BID). (2006). Las políticas de las políticas públicas. Progreso económico y social en América Latina. David Rockefeller Center for Latin American Studies, Harvard University. Recuperado de: http://idbdocs.iadb.org/wsdocs/getdocument. aspx?docnum $=912428$

Consejo Superior de Educación (CSE). (2008). Acta Ordinaria No. 51-2008 del Consejo Superior de Educación. Recuperado de: http://cse.go.cr/sites/default/files/acuerdos/acta 51-2008 normas de promocion y repitencia.pdf 
Contraloría General de la República. (2011). Informe N. DFOE-SOC-IF-13-2011. Recuperado de: http://www.asamblea.go.cr/Informes de la Contraloria/Informes\%202011/k-\%20 informes noviembre 2011/DFOE-SOC-IF-13-2011.pdf

Holguín, C. (2013). La participación social y ciudadanía en las políticas públicas: apuntes para su reflexión. En: Roth D., A. (ed). El análisis y la evaluación de las políticas públicas en la era de la participación. Reflexiones teóricas y estudios de casos. Colombia: Universidad Nacional de Colombia.

Merino, M. (2013). Políticas públicas. Ensayo sobre la intervención del Estado en la solución de problemas públicos. México: CIDE.

Instituto Nacional de Estadísticas y Censos (INEC). (2013). Estadísticas pobreza. San José, Costa Rica: Autor.

Ministerio de Educación Pública (MEP). (2011). Consideraciones respecto a las normas reguladoras de la promoción y la repitencia dentro del sistema público costarricense. San José, Costa Rica: Autor.

Picado, C. y Abarca, A. (2012). La política pública en promoción y repitencia en la Educación Secundaria, Costa Rica. Anuario Centro de Investigaciones y Estudios Políticos, 3, 126-147. Recuperado de: http://revistas.ucr.ac.cr/index.php/ciep/article/view/14644/13901

Programa Estado de la Nación. (2011). Tercer Informe del Estado de la Educación. Megabases de datos georreferenciados para la educación primaria y secundaria de los centros educativos de Costa Rica (2000-2009). Programa Estado de la Nación. San José, Costa Rica: CONAREESTADO DE LA NACIÓN. 